\title{
PEGylated PLGA nanospheres optimized by DoE for ocular administration of dexibuprofen - in vitro, ex vivo and in vivo characterization
}

Authors: Sánchez-López E. ${ }^{1,2}$, Egea M.A. ${ }^{1,2}$, Cano A. ${ }^{1}$, Espina M. ${ }^{1,2}$, Calpena A.C. ${ }^{2,3}$, Ettcheto M. ${ }^{4,5}$, Camins A. ${ }^{4,5}$, Souto E.B. ${ }^{6,7}$, Silva A.M. ${ }^{8,9}$, García M.L. ${ }^{1,2}$

${ }^{1}$ Department of Physical Chemistry, Faculty of Pharmacy, University of Barcelona, Barcelona 08028, Spain.

${ }^{2}$ Institute of Nanoscience and nanotechnology (IN2UB). Faculty of Pharmacy, University of Barcelona, Barcelona 08028, Spain.

${ }^{3}$ Department of Biopharmacy and Pharmaceutical Technology, Faculty of Pharmacy, University of Barcelona, Barcelona 08028, Spain.

${ }^{4}$ Department of Department of Pharmacology and Therapeutic Chemistry. Faculty of Pharmacy, University of Barcelona, Barcelona 08028, Spain.

${ }^{5}$ Centro de Investigación Biomédica en Red de Enfermedades Neurodegenerativas (CIBERNED), University of Barcelona, Barcelona 08028, Spain.

${ }^{6}$ Department of Pharmaceutical Technology, Faculty of Pharmacy, University of Coimbra (FFUC), Polo das Ciências da Saúde, Azinhaga de Santa Comba, 3000-548 Coimbra, Portugal.

${ }^{7} \mathrm{CNC}$ - Center for Neuroscience and Cell Biology, University of Coimbra (FFUC), Coimbra, Portugal.

${ }^{8}$ Department of Biology and Environment, School of Life and Environmental Sciences, (ECVA, UTAD), University of Trás-os-Montes and Alto Douro, Quinta de Prados, Vila Real 5001-801, Portugal

${ }^{9}$ Centre for the Research and Technology of Agro-Environmental and Biological Sciences, University of Trás-os-Montes and Alto Douro, CITAB-UTAD, Vila-Real 5001-801, Portugal

Corresponding author: M. Luisa. García. Department of Physical Chemistry, Faculty of Pharmacy, University of Barcelona, Barcelona, 08028, Spain. Phone: +34 9340245 52. Fax: +34 934035 987. marisagarcia@ub.edu

\section{ABSTRACT}

Dexibuprofen-loaded PEGylated PLGA nanospheres have been developed to improve the biopharmaceutical profile of the anti-inflammatory drug for ocular administration. Dexibuprofen is the active enantiomer of ibuprofen and therefore lower doses may be applied to achieve the same therapeutic level. According to this, two batches of nanospheres of different drug concentrations, 0.5 and $1.0 \mathrm{mg} / \mathrm{ml}$ respectively have been developed (the last one corresponding to the therapeutic ibuprofen concentration for inflammatory eye diseases). Both batches were composed of negatively charged nanospheres (-14.1 and $-15.9 \mathrm{mV}$ ), with a mean particle size below $200 \mathrm{~nm}$, and a high encapsulation efficiency (99\%). X-ray, FTIR, and DSC analyses confirmed that the drug was dispersed inside the matrix of the nanospheres. While the in vitro release profile was sustained up to 12 hours, the ex vivo corneal and scleral permeation profile demonstrated higher drug retention and permeation in the corneal tissue rather than in the sclera. These results were also confirmed by the quantification of dexibuprofen in ocular tissues after the in vivo administration of drug-loaded nanospheres. Cell viability studies confirmed that PEGylated-PLGA nanospheres were less cytotoxic than free dexibuprofen in the majority of the tested concentrations. Ocular in vitro (HET-CAM test) and in vivo (Draize test) tolerance assays demonstrated the non-irritant character of both nanosphere batches. 
47 In vivo anti-inflammatory effects were evaluated in albino rabbits before and after 48 inflammation induction. Both batches confirmed to be effective to treat and prevent ocular 49 inflammation.

Keywords: Nanospheres; Dexibuprofen; PLGA; PEG; Inflammation; Drug Delivery.

\section{Chemical compounds studied in this article}

54 Lactic acid (PubChem CID: 612); Glycolic acid (PubChem CID: 757); Ethylene glycol 55 (PubChem CID: 174); Dexibuprofen (PubChem CID: 39912); Polyvinyl alcohol 56 (PubChem CID: 11199);

\section{Abbreviations}

59 NSAIDs, non-steroidal anti-inflammatory drugs; IBU, ibuprofen; DXI, dexibuprofen; GI, 60 gastrointestinal; NPs, nanoparticles; PLGA, poly(lactic-co-glycolic) acid; PEG, 61 poly(ethylene glycol); RES, reticuloendothelial system; NSs, nanospheres; PVA, 62 polyvinyl alcohol; DoE, design of experiments; $Z_{\mathrm{av}}$, average particles size; $\mathrm{PI}$, 63 polydispersity index; ZP, zeta potential; EE, encapsulation efficiency; PCS, photon 64 correlation spectroscopy; HPLC, high performance liquid chromatography; TEM, 65 transmission electron microscopy; DSC, differential scanning calorimetry; XRD, X-Ray 66 diffraction; FTIR, Fourier transformed infrared; PBS, phosphate buffered saline; BR, 67 bicarbonate ringer; CAM, chorioallantoic membrane; SA, sodium arachidonate. 


\section{Introduction}

Inflammation is a non-specific response of the body against injuries from the external environment, acting as a defense mechanism to isolate and destroy the triggering agent, as well as to repair the damaged tissues. Ocular inflammation is one of the most prevalent diseases in ophthalmology. It can affect any part of the eye or the surrounding tissues. Corticosteroids are commonly used as anti-inflammatory drugs in the treatment of ocular inflammation but they induce serious adverse effects when administered continuously [1]. The main alternatives to corticosteroids in the treatment of inflammation are non-steroidal anti-inflammatory drugs (NSAIDs) [2]. In the field of ophthalmology, Ibuprofen (IBU) has been receiving particular attention in recent years due to its anti-inflammatory activity, having however a number of adverse effects that limit its use [3].

Rapid elimination of NSAIDs administered as eye drops, results in a pre-corneal drug half-life between 1-3 min. As a consequence, only a very small amount of the drug (1$5 \%$ of the dose) actually penetrates the cornea and is able to reach intraocular tissues. On the other hand, drugs administered onto the ocular mucosa are known to suffer absorption via conjunctiva and nasolacrimal duct, easily reaching the systemic circulation [4] and [5]. Drugs, such as ibuprofen, may induce adverse side effects that can be minimized by the use of the active enantiomer - dexibuprofen (DXI), which is twice more potent and has less side effects than the former [6]. Gastric and epigastric pain, nausea and vomiting have been the most frequent side effects reported in randomized clinical trials in patients treated with DXI. Effects of DXI in the central nervous system (CNS) were less common than the use of racemic ibuprofen [7]. The racemic mixture was also responsible for a higher gastric toxicity than the $\mathrm{S}(+)$ isomer [6]. Moreover, the safety, tolerability and equivalent efficacy between DXI and the double dose of ibuprofen was confirmed by comparing the oral uptake of both drugs for osteoarthritis treatment in a clinical study [7] and [8].

To protect the drug from inactivation by the enzymes present in the tear film or corneal epithelium, to facilitate its transcorneal penetration prolonging its stay in the precorneal area, and to avoid undesired adverse effects, polymeric nanoparticles (NPs) have been proposed. Biodegradable polymers, including poly(lactic-co-glycolic acid) (PLGA) (biopolymer approved by the Food and Drug administration), have been widely used as a biomaterial in medical prostheses and surgical sutures [9]. More recently, PLGA has been used in the development of colloidal carriers for controlled release of drugs, due to its biocompatibility, biodegradability and non-toxicity [10]. Furthermore, compared to natural polymers, these synthetic polymers demonstrate higher reproducibility, are easily formulated and allow the control and prediction of the degradation kinetics [11].

Among other, strategies, PEG-coating on PLGA NPs offer several advantages. These are firstly attributed to the enhanced contact time of the particles with the corneal surface by the interaction with the mucus layer of the tear film. NPs interact with the mucus layer of the tear film either by electrostatic, hydrophobic and hydrogen bonding, or by their physical retention in the mucin network [12]. Griffiths et al. [12] demonstrated that such retention in the mucin network is dependent on the hydrophobic surface of the particles, which could be overcome by coating them with PEG. On the other hand, the hydrophobic 
entrapment could be minimized as long as the nanoparticles were adequately surfaced with such hydrophilic PEG layers and depicted negative electrical charge [12]. Therefore, the accumulation of the NPs in the conjunctival sac, as well as, the ability of the particles to penetrate in the first layers of the corneal epithelium contribute to enhance drugs' bioavailability [13]. In addition, PEGylation contributes to maintain the particles in circulation for a longer time, thus avoiding their recognition by the reticuloendothelial system (RES) [14].

In the present work, we report the development of a new formulation for ocular delivery of dexibuprofen (DXI), based on nanospheres (NSs) composed of poly-L-lactic-coglycolide (PLGA) surrounded by polyethylene glycol (PEG) chains (DXI-PLGA-PEG NSs). The suitability of DXI-PLGA-PEG NSs to treat and prevent ocular inflammation has been demonstrated. Physicochemical properties and drug-polymer interactions were assessed. In vitro and ex vivo drug release and short-term stability of DXI NSs were studied. DXI quantification after in vivo administration was also performed.

\section{Materials and methods}

\subsection{Materials}

Diblock copolymer PLGA-PEG 5\% Resomer ${ }^{\circledR}$ was obtained from Evonik Corporation (Birmingham, USA) and the active compound $S$-(+)-Ibuprofen (dexibuprofen) was purchased from Amadis Chemical (Hangzou, China). Polyvinyl alcohol (PVA) and acetone were purchased from Sigma-Aldrich (Madrid, Spain) and Fisher Scientific (Pittsburgh, USA), respectively. Reagents for cell culture were obtained from Gibco (Alfagene, Portugal). Alamar Blue, from Invitrogen Alfagene ${ }^{\circledR}$ (Portugal), was used for cell viability estimation. Water filtered through Millipore MilliQ system was used for all the experiments and all the other reagents were of analytical grade.

\subsection{Methods}

\subsubsection{Nanospheres preparation}

NSs were prepared by solvent displacement method described elsewhere [15]. Briefly, the co-polymer PLGA-PEG and the drug (DXI) were firstly dissolved in acetone. This organic phase was added dropwise, under moderate stirring, into $10 \mathrm{ml}$ of an aqueous solution of PVA (0.33-1.17 \%) adjusted to the desired $\mathrm{pH}$ (3.2-4.8). After that, acetone was evaporated under reduced pressure and the resulting particles were ultracentrifuged, at 15000 r.p.m. for $20 \mathrm{~min}$, in order to remove excess of PVA.

\subsubsection{Optimization of nanospheres parameters}

Design of experiments (DoE) is frequently used to plan research because it provides maximum information, whilst requiring a minimal number of experiments [16]. A central composite factorial design was developed to analyze the effect of independent variables ( $\mathrm{pH}, \mathrm{DXI}$ and PVA concentrations) on the dependent variables (average particle size 
$\left(\mathrm{Z}_{\mathrm{av}}\right)$, polydispersity index $(\mathrm{PI})$, zeta potential $(\mathrm{ZP})$ and encapsulation efficiency $(\mathrm{EE})$. The amount of polymer was kept constant for all the assays (90 mg).

According to the composite design matrix generated by Statgraphics Plus 5.1 software, a total of 16 experiments ( 8 factorial points, 6 axial points and two replicated center points) were required. The experimental responses were the result of the individual influence and the interactions of the three independent variables, as shown in Table 1. The responses were therefore modeled through the full second-order polynomial equation shown in equation $/ 1 /$ : /1/

where $Y_{u}$ is the measured response, $\beta_{0}$ to $\beta_{2,3}$ are the regression coefficients and $X_{1}, X_{2}$ and $\mathrm{X}_{3}$ are the studied factors. The effect and the significance level of the factors were evaluated by analysis of variance (ANOVA) [17].

Table 1. Values of the experimental factors according to the matrix designed by $2^{3}+$ star 172

\begin{tabular}{|c|c|c|c|c|c|c|c|c|c|c|}
\hline & \multicolumn{2}{|c|}{$\mathrm{pH}$} & \multicolumn{2}{|c|}{$\mathrm{c}_{\mathrm{DXI}}$} & \multicolumn{2}{|c|}{$\mathrm{c}_{\mathrm{PVA}}$} & \multirow[b]{2}{*}{$\mathrm{Z}_{\mathrm{av}}(\mathrm{nm})$} & \multirow[b]{2}{*}{ PI } & \multirow[b]{2}{*}{$\mathrm{ZP}(\mathrm{mV})$} & \multirow[b]{2}{*}{$\mathrm{EE}(\%)$} \\
\hline & $\begin{array}{c}\text { Coded } \\
\text { level }\end{array}$ & $\mathrm{pH}$ & $\begin{array}{c}\text { Coded } \\
\text { level }\end{array}$ & $(\mathrm{mg} / \mathrm{ml})$ & $\begin{array}{c}\text { Coded } \\
\text { level }\end{array}$ & $(\%)$ & & & & \\
\hline \multicolumn{11}{|c|}{\begin{tabular}{|l|} 
Factorial points \\
\end{tabular}} \\
\hline F1 & -1 & 3.5 & -1 & 0.50 & -1 & 0.50 & $221.4 \pm 0.5$ & $\mathbf{0 . 0 8 2} \pm 0.023$ & $-\mathbf{- 4 . 1 7} \pm 0.29$ & 99.79 \\
\hline $\mathrm{F} 2$ & 1 & 4.5 & -1 & 0.50 & -1 & 0.50 & $219.3 \pm 6.6$ & $0.050 \pm 0.033$ & $-11.9 \pm 0.19$ & 90.80 \\
\hline $\mathrm{F} 3$ & -1 & 3.5 & 1 & 1.50 & -1 & 0.50 & $225.5 \pm 3.2$ & $0.072 \pm 0.027$ & $-3.16 \pm 0.28$ & 99.20 \\
\hline $\mathrm{F} 4$ & 1 & 4.5 & 1 & 1.50 & -1 & 0.50 & $201.1 \pm 4.6$ & $0.048 \pm 0.018$ & $-5.13 \pm 0.55$ & 87.65 \\
\hline F5 & -1 & 3.5 & -1 & 0.50 & 1 & 1.00 & $216.5 \pm 2.9$ & $0.068 \pm 0.026$ & $-3.24 \pm 0.93$ & 90.72 \\
\hline F6 & 1 & 4.5 & -1 & 0.50 & 1 & 1.00 & $216.6 \pm 2.1$ & $0.049 \pm 0.007$ & $-5.65 \pm 0.26$ & 85.52 \\
\hline F7 & -1 & 3.5 & 1 & 1.50 & 1 & 1.00 & $219.0 \pm 3.8$ & $0.065 \pm 0.009$ & $-3.53 \pm 0.17$ & 89.77 \\
\hline F8 & 1 & 4.5 & 1 & 1.50 & 1 & 1.00 & $213.3 \pm 1.2$ & $0.048 \pm 0.029$ & $-3.15 \pm 0.53$ & 89.82 \\
\hline
\end{tabular}

Axial points

\begin{tabular}{|c|c|c|c|c|c|c|c|c|c|c|}
\hline F9 & 1.68 & 4.8 & 0 & 1.00 & 0 & 0.75 & $229.2 \pm 0.7$ & $0.054 \pm 0.018$ & $-2.75 \pm 0.17$ & 89.87 \\
\hline F10 & -1.68 & 3.2 & 0 & 1.00 & 0 & 0.75 & $205.8 \pm 2.3$ & $0.063 \pm 0.013$ & $-2.53 \pm 0.44$ & 92.36 \\
\hline F11 & 0 & 4.0 & 1.68 & 1.84 & 0 & 0.75 & $223.5 \pm 0.6$ & $0.036 \pm 0.018$ & $-4.38 \pm 0.25$ & 90.90 \\
\hline F12 & 0 & 4.0 & -1.68 & 0.16 & 0 & 0.75 & $223.4 \pm 1.6$ & $0.052 \pm 0.022$ & $-6.84 \pm 0.27$ & 99.10 \\
\hline F13 & 0 & 4.0 & 0 & 1.00 & 1.68 & 1.17 & $220.4 \pm 0.3$ & $0.036 \pm 0.007$ & $-5.73 \pm 0.13$ & 98.90 \\
\hline F14 & 0 & 4.0 & 0 & 1.00 & -1.68 & 0.33 & $203.9 \pm 0.4$ & $0.072 \pm 0.024$ & $-8.18 \pm 0.23$ & 97.84 \\
\hline
\end{tabular}

Center points

\begin{tabular}{|l|l|l|l|l|l|l|l|l|l|l|}
\hline F15 & 0 & 4.0 & 0 & 1.00 & 0 & 0.75 & $220.3 \pm 6.6$ & $0.046 \pm 0.023$ & $-6.01 \pm 0.16$ & 90.51 \\
\hline F16 & 0 & 4.0 & 0 & 1.00 & 0 & 0.75 & $217.3 \pm 2.9$ & $0.043 \pm 0.030$ & $-6.56 \pm 0.93$ & 85.45 \\
\hline
\end{tabular}

173

\subsubsection{Physicochemical characterization}

$\mathrm{Z}_{\mathrm{av}}$ and PI of NSs were determined by photon correlation spectroscopy (PCS) (after 1:10 dilution) with a Zetasizer Nano ZS (Malvern Instruments, Malvern, UK) at $25{ }^{\circ} \mathrm{C}$ using disposable quartz cells and (Malvern Instruments). 
NSs surface charge, measured as zeta potential (ZP), was evaluated by using laserDoppler electrophoresis with M3 PALS system in Zetasizer Nano ZS. ZP indirectly indicates the rate of aggregation of particles. A greater ZP (in absolute value) would induce less aggregation due to repulsion forces between the particles. To calculate this, the Henry equation was used /2/:

$$
\mu_{\mathrm{E}}=\frac{\varepsilon \mathrm{ZPf}(\mathrm{Ka})}{6 \pi \eta}
$$

where $\mu_{\mathrm{E}}$ is the electrophoretic mobility, $\varepsilon$ is the dielectric constant of the medium, ZP is the zeta potential, $\eta$ is the viscosity of the medium, $K$ is the Deybye-Hückel parameter and $\mathrm{f}(\mathrm{Ka})$ is a correction factor that takes into account the thickness of the electrical double layer $(1 / \mathrm{K})$ and particle diameter (a). The unit of $\mathrm{K}$ is a reciprocal length.

The reported values correspond to the mean \pm SD of at least three different batches of each formulation. [18].

\subsubsection{Evaluation of the encapsulation efficiency}

The EE of DXI in the NSs was determined indirectly by measuring the concentration of the free drug in the dispersion medium. The non-encapsulated DXI was separated by a filtration/centrifugation technique (1:10 dilution) by using an Ultracell-100K (AmiconR Ultra; Millipore Corporation, Massachusetts) centrifugal filter devices at $4{ }^{\circ} \mathrm{C}$ and $700 \mathrm{~g}$ for 30 min (Heraeus, Multifuge $3 \mathrm{~L}-\mathrm{R}$, Centrifuge. Osterode, Germany). The EE was calculated using equation $/ 3 /$ :

$$
\mathrm{EE}(\%)=\frac{\text { total amount of } D X I-\text { free } D X I}{\text { total amount of } D X I} \times 100
$$

Samples were evaluated by high performance liquid chromatography (HPLC), as described elsewhere [19]. Briefly, samples were quantified using HPLC Waters 2695 separation module and a Kromasil ${ }^{\circledR} \mathrm{C}_{18}$ column $(5 \mu \mathrm{m}, 150 \times 4.6 \mathrm{~mm})$ with a mobile phase of methanol/ phosphoric acid $0.05 \%(80: 20)$ at a flow rate of $1 \mathrm{ml} / \mathrm{min}$ and a wavelength of $220 \mathrm{~nm}$. Standards were prepared in methanol:water (90:10) from a stock solution of $500 \mu \mathrm{g} / \mathrm{ml}(50-0.5 \mu \mathrm{g} / \mathrm{ml})$. Data was processed using Empower $3^{\circledR}$ Software.

\subsubsection{Nanospheres characterization and interaction studies}

NSs were diluted (1:5) and a morphological study was carried out by transmission electron microscopy (TEM) on a Jeol 1010. To visualize the NSs, copper grids were activated with UV light and samples were placed on the grid surface. Negative staining was performed with uranyl acetate (2\%).

X-ray diffraction (XRD) was used to analyze the state (amorphous or crystalline) of the samples (centrifuged NSs or formulation compounds). Compounds were sandwiched between polyester films and exposed to CuK" radiation $(45 \mathrm{kV}, 40 \mathrm{~mA}, \lambda=1.5418 \AA)$ in

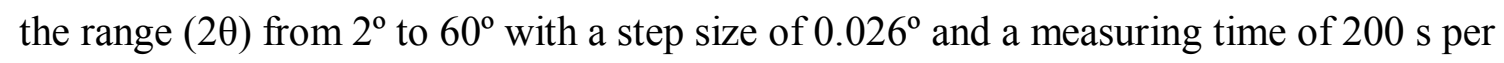
step. 
Fourier transform infrared (FTIR) spectra of different samples (NSs formulations or compounds separately) were obtained using a Thermo Scientific Nicolet iZ10 with an ATR diamond and DTGS detector. The scanning range was $525-4000 \mathrm{~cm}^{-1}$. Thermograms were obtained on a Mettler TA 4000 system (Greifensee, Switzerland) equipped with a DSC 25 cell. Temperature was calibrated by the melting transition point of indium prior to sample analysis. All samples were weighed (Mettler M3 Microbalance) directly in perforated aluminum pans and heated under a nitrogen flow at a rate of 10 ${ }^{\circ} \mathrm{C} / \min \left(25-125^{\circ} \mathrm{C}\right)$.

\subsubsection{Determination of the in vitro release profile}

One of the main goals of drug release from the polymer matrix is the possibility to provide an extended release profile over time. In vitro release was evaluated using a bulkequilibrium reverse dialysis bag technique [20]. This technique is based on the dispersion of the colloidal suspension in the dialysis medium accomplishing sink conditions [21]. The release medium was composed of a buffer solution (PBS 0.1 M, pH 7.4). 16 dialysis sacs containing $1 \mathrm{ml}$ of PBS were previously immersed into the release medium. The dialysis sacs were equilibrated with the dissolution medium a few hours prior to the experiments. A volume of $15 \mathrm{ml}$ of free drug in PBS or NSs was added to $285 \mathrm{ml}$ of the dissolution medium. The assay was carried out in triplicate comparing the free drug in PBS against NSs formulations. Release kinetic experiments were performed at a fixed temperature of $32^{\circ} \mathrm{C}$ (ocular surface temperature) under constant magnetic stirring ( $n=6 /$ group). At predetermined time intervals, the dialysis sacs were withdrawn from the stirred release solution and the volume was replaced by $1 \mathrm{ml}$ of PBS. The content of the sacs at each time point was evaluated and data were adjusted to the most common kinetic models [18].

\subsubsection{Ex vivo corneal and scleral permeation study}

Ex vivo corneal and scleral permeation experiments were carried out with New Zealand rabbits (male, weighting 2.5-3.0 kg), under veterinary supervision, and according to the Ethics Committee of Animals Experimentation from the University of Barcelona (CEEAUB). The rabbits were anesthetized with intramuscular administration of ketamine $\mathrm{HCl}$ (35 mg/kg) and xylazine $(5 \mathrm{mg} / \mathrm{kg})$ and euthanized by an overdose of sodium pentobarbital $(100 \mathrm{mg} / \mathrm{kg})$ administered through marginal ear vein under deep anesthesia. The cornea and sclera were excised and immediately transported to the laboratory in artificial tear solution. The assay was done using Franz diffusion cells and the tissue was fixed between the donor and receptor compartment. The area available for permeation was $0.64 \mathrm{~cm}^{2}$. The receptor compartment was filled with freshly prepared Bicarbonate Ringer's (BR) solution. This compartment was kept at 32 and $37 \pm 0.5^{\circ} \mathrm{C}$ for corneal and scleral permeation, respectively, and stirred continuously. A volume of $1 \mathrm{~mL}$ of $\mathrm{F}$ (A) $\mathrm{NSs}$ or $0.5 \mathrm{mg} / \mathrm{ml}$ of DXI was placed in the donor compartment and covered to avoid evaporation. A volume of $300 \mu \mathrm{l}$ was withdrawn from the receptor compartment at fixed times and replaced by an equivalent volume of fresh BR solution at the same temperature. 
The cumulative DXI amount permeated was calculated, at each time point, from DXI amount in the receiving medium and plotted as function time (min) [22].

At the end of the study, the cornea was used to determine the amount of drug retained. The tissue was cleaned using a $0.05 \%$ solution of sodium lauryl sulfate and washed with distilled water, weighed and treated with methanol under sonication during $30 \mathrm{~min}$ using an ultrasound bath. The amount of DXI permeated and retained through the cornea was determined.

Results are reported as the median \pm SD of six replicates for the amount of DXI permeated and retained on each tissue, respectively [22].

Lag time, $T_{L}(h)$, values were calculated by plotting the cumulative DXI permeating the cornea versus time, determining $\mathrm{x}$-intercept by linear regression analysis. The corneal permeability coefficient $K_{P}(\mathrm{~cm} / h)$, partition coefficient $\mathrm{P}_{1}(\mathrm{~cm})$ and diffusion coefficient $\mathrm{P}_{2}\left(\mathrm{~h}^{-1}\right)$ were calculated from the following equations:

$K_{p}=P_{1} \times P_{2}$

$P_{1}=\frac{J}{A \times C_{0} \times P_{2}}$

$P_{2}=\frac{1}{6 \times T_{L}}$

$/ 6 /$

where $\mathrm{C}_{0}$ is the initial concentration of drug in the donor compartment, $\mathrm{A}\left(0.64 \mathrm{~cm}^{2}\right)$ is the exposed corneal surface [22].

\subsubsection{Short-term stability}

NSs stability at 4,25 and $38^{\circ} \mathrm{C}$ was assessed by light backscattering by means of a Turbiscan ${ }^{\circledR}$ Lab. For this purpose, a glass measurement cell was filled with the sample for each temperature. The light source, pulsed near infrared light-emitting diode LED $(\lambda=880$ $\mathrm{nm}$ ), was received by a backscattering detector at an angle of $45^{\circ}$ from the incident beam. Backscattering data were acquired once a month for $24 \mathrm{~h}$, at $1 \mathrm{~h}$ intervals. In addition to this technique, NSs $\mathrm{Z}_{\mathrm{av}}$, PI and ZP were also measured monthly. Temperature studies were carried out by duplicate and visual observation of the samples was undertaken.

\subsubsection{Cytotoxicity assay}

Alamar blue assay was carried out in order to investigate the possible toxicity of the developed NSs in comparison with free DXI. To perform this assay Y-79 (human retinoblastoma) cell line acquired from Cell Lines Service (CLS, Eppelheim, Germany) was used. Y-79 cells were maintained in RPMI-1640, supplemented with $10 \%$ (v/v) fetal bovine serum (FBS), $2 \mathrm{mM} \mathrm{L-glutamine,} \mathrm{and} \mathrm{antibiotics} \mathrm{(100} \mathrm{U} / \mathrm{ml}$ penicillin and $100 \mu \mathrm{g}$ $\mathrm{mL}^{-1}$ of streptomycin) at $37{ }^{\circ} \mathrm{C}$ under an atmosphere of $5 \% \mathrm{CO}_{2} / 95 \%$ air with controlled humidity (Binder chamber). Cells were centrifuged, re-suspended in FBS-free culture media, counted and seeded, after appropriate dilution, at $1 \times 10^{5}$ cells $/ \mathrm{ml}$, in poly-L-lysine pre-coated 96-well plates (100 $\mu \mathrm{l} /$ well). For this study, dilutions of NSs in FBS-free culture media (namely $\mathrm{F}(\mathrm{A})$ and $\mathrm{F}(\mathrm{B})$, see NSs optimization section), as well as their corresponding free drug were carried out and added to cells $24 \mathrm{~h}$ after seeding (100 
$\mu \mathrm{L} /$ well). Cell viability was assayed with Alamar Blue ( $\mathrm{BB}$, Alfagene, Invitrogen, Portugal), 24 or $48 \mathrm{~h}$ after exposure to test compounds, by addition of $100 \mu \mathrm{l} /$ well of $\mathrm{AB}$ solution, $10 \%(\mathrm{v} / \mathrm{v})$ diluted in FBS-free media, preceded by removal of test solutions. The $\mathrm{AB}$ absorbance was determined at $\lambda$ of $570 \mathrm{~nm}$ (reduced form) and $620 \mathrm{~nm}$ (oxidized form) after $4 \mathrm{~h}$ of cell contact. Data were analyzed by calculating the percentage of Alamar blue reduction (according to the manufacture recommendations) and expressed as percentage of control (untreated), as reported before [23].

\subsubsection{Ocular tolerance assays: HET-CAM and Draize irritation test}

To assess the potential risk of ocular irritation caused by NSs, ocular tolerance test by in vivo and in vitro methods were carried out.

To study the ocular tolerance in vitro the HETCAM ${ }^{\circledR}$ test was developed as described in the INVITTOX $n^{\circ} 15$ protocol [24]. This test is based on the observation of the irritant effects (bleeding, vasoconstriction and coagulation) in the chorioallantoic membrane (CAM) of an embryonated egg (10 days) induced by application of $300 \mu$ l of the studied formulation, during the first 5 minutes [25]. In the experimental procedure, fertilized and incubated eggs during 10 days were used. These eggs (from the farm G.A.L.L.S.A, Tarragona, Spain) were maintained at a temperature of $12 \pm 1^{\circ} \mathrm{C}$ for at least 24 hours before placing them in the incubator with controlled temperature $\left(37.8^{\circ} \mathrm{C}\right)$ and humidity (50-60\%) during the incubation days. A series of controls were performed: SDS 1\% (positive control for slow irritation), $0.1 \mathrm{~N} \mathrm{NaOH}$ (positive control for fast irritation), $\mathrm{NaCl} 0.9 \%$ (negative control). Data were analyzed as the media $\pm \mathrm{SD}$ of the time at which the injury occurred ( $n=6 /$ group). Scores of irritation potential can be grouped into four categories (see Table A.1 of Supplementary Material) [26].

In vivo ocular tolerance assays were performed using primary eye irritation test of Draize et al. [22] using New Zealand albino male rabbits of $2.5 \mathrm{~kg}$ middle weight from San Bernardo farm (Navarra). This test was performed according to the Ethical Committee for Animal Experimentation of the UB and current legislation (Decret 214/97, Gencat). The sample was placed in the conjunctival sac of the right eye and a gentle massage was applied to assure the proper sample circulation through the eye. The appearance of irritation was observed at the time of administration and after 1 hour, using the left eye as a negative control ( $n=6 /$ group). The evaluation was performed by direct observation of the anterior segment of the eye, noting the possible injury of the conjunctiva (inflammation, chemosis, redness or oozing), iris and cornea (opacity and affected surface) (for detailed punctuation see Table A.2 of Supplementary Material). Ocular irritation index (OII) was evaluated according to the observed injuries (Table A.1 on Supplementary material).

\subsubsection{Inflammatory activity assay}


Corneal inflammatory activity of the developed formulations was assessed in vivo ( $n=6 /$ group). Ocular inflammation was induced administering $50 \mu \mathrm{l}$ of sodium arachidonate (SA) $0.5 \%(\mathrm{w} / \mathrm{v})$ dissolved in PBS ( $\mathrm{pH} 7.4)$. Inflammation was quantified using a slit lamp at various times, according to a modified Draize scoring system [26]. The sum of the inflammation score is expressed by the mean \pm SD (detailed punctuations can be found in Table A.2 of supplementary Material).

To assess inflammation prevention, free drug and DXI NSs were instilled (50 $\mu \mathrm{l})$ in the conjunctival sac, $30 \mathrm{~min}$ before induction of ocular inflammation. In order to test inflammation treatment, ocular inflammation was induced and after $30 \mathrm{~min}$, either NSs or free DXI in saline serum were applied.

\subsubsection{Ocular drug bioavailability}

In order to achieve steady-state concentrations, DXI NSs were administered to New Zealand rabbits $(n=6)$, every 8 hours for two weeks . A volume of $50 \mu$ l of each formulation was administered and, at the end of the experiments, animals were scarified and drug amount was quantified in vitreous humor and aqueous humor. Retained DXI on cornea and sclera were also measured. [19].

\subsubsection{Statistical analysis}

All of the data are presented as the mean \pm S.D. Two-way ANOVA followed by Tukey post hoc test was used for multi-group comparison. Student's $t$-test was used for twogroup comparisons. Statistical significance was set at $p<0.05$. GraphPad Prism V6.0 InStat (GraphPad Sofware Inc., San Diego, CA, EE.UU.) was used to carry out the analysis.

\section{Results and discussion}

\subsection{Nanospheres optimization}

The results obtained from the central composite factorial design are shown in Table 1. EE is greatly influenced by $\mathrm{pH}$ (Figure 1a) and decreases at alkaline media. Therefore, a low $\mathrm{pH}$ value would have to be chosen. Moreover, this acidic media would contribute to obtain a monodisperse population, as the alkaline $\mathrm{pH}$ values were shown to increase PI (Figure 1b). However, acidic $\mathrm{pH}$ contributes to sample instability by decreasing the ZP in absolute values (Figure A.1 of Supplementary Material). In order to obtain a balance between the long-term stability of the particles and the physicochemical NSs parameters,a $\mathrm{pH}$ of 3.5 was selected (F1, Table 1).

The increase of DXI concentration in the formulations did not have a significant effect on the EE, suggesting that the tested concentrations did not reach polymer-loading capacity. Further studies with F1 particles were carried out, leading to a high EE (99\%) using $45 \mathrm{mg}$ of PLGA-PEG. Drug loading capacity depends on the physicochemical 
properties of the molecule as well as of the nanoparticle polymer, and also on the manufacturing process for these nanoparticle systems [27]. In our case, despite the small drug concentration in F1 $(0.5 \mathrm{mg} / \mathrm{ml})$, some authors have suggested that DXI is more effective than the racemic counterpart (ibuprofen) in a ratio 1:0.5 [3]. Thus, this concentration would theoretically be enough to treat corneal inflammation. A second formulation, containing an identical drug/polymer ratio but twice the amount of the drug $(1 \mathrm{mg} / \mathrm{ml})$, was also developed and characterized for storage stability, inflammation and irritation assays. Both formulations have been studied: F(A) for NSs containing DXI 0.5 $\mathrm{mg} / \mathrm{ml}$, and $45 \mathrm{mg}$ of polymer and F(B) for NSs containing $1 \mathrm{mg} / \mathrm{ml} \mathrm{DXI} \mathrm{and} 90 \mathrm{mg}$ of polymer.

(a)

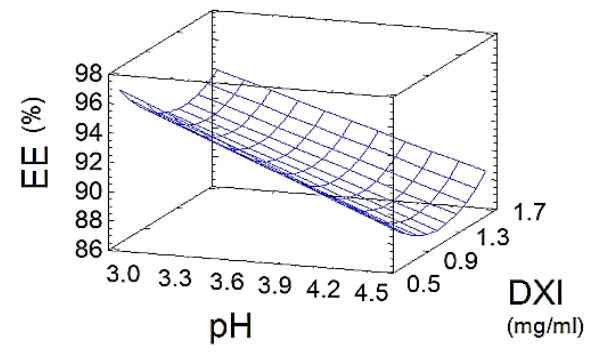

(b)

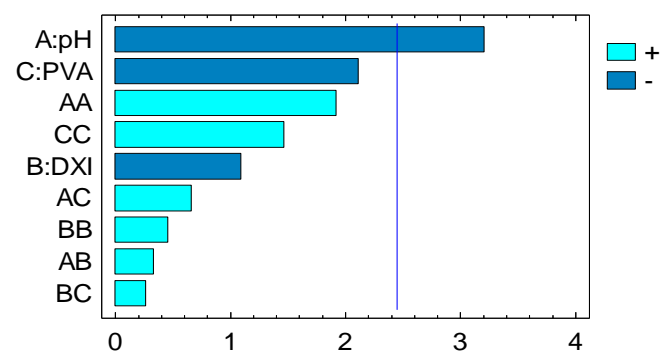

Figure 1. Optimization of DXI NSs. (a) EE (\%) surface response at a fix PVA concentration $(0.75 \%)$, (b) PI surface response at a fix DXI concentration $(0.5 \mathrm{mg} / \mathrm{ml})$.

\subsection{Nanospheres characterization and interaction studies}

NSs parameters after ultracentrifugation are summarized in Table A.3 (Supplementary Material). Both formulations presented a monodisperse population $(\mathrm{PI}<0.1)$ and a mean size below $200 \mathrm{~nm}-$, suitable for ocular administration. Superficial charge was negative (around $-15 \mathrm{mV}$ ) due to polymer carboxylic chains [28]. The observed decrease on the ZP values, compared to those reported for PLGA-NPs by Vega et al. [26] (higher than $-20 \mathrm{mV}$ ), attributed to the presence of PEG layer, which reduces the negative surface charge characteristic of PLGA-NPs. The carboxylic groups of PLGA were probably masked by PEG due to nanoparticles production by solvent displacement technique. In this method, a microphase separation occurs because of PLGA and PEG mutual immiscibility. PLGA backbone would collapse easily in water (non-solvent for PLGA), leaving the PEG chains toward the external surface of the emulsion droplets facing the aqueous phase (good solvent for PEG) [29].

TEM images (Figure A.2, Supplementary Material) reveal that the optimized NSs showed spherical shape without signals of aggregation phenomena. The mean NSs size was similar to that obtained by PCS $(<200 \mathrm{~nm})$.

In order to study interactions between drug and NSs, spectroscopic analysis and DSC studies were carried out.

XRD profiles of DXI (Figure 2a) show sharp intense peaks of crystallinity whereas the polymer diffracted an amorphous pattern. PVA exhibits a peak at $2 \theta=20^{\circ}$ due to its 
426 semi-crystalline state. Empty NSs, drug loaded NSs and PLGA-PEG present similar 427 profiles. The peaks corresponding to the drug were not detected in DXI NSs. This may 428 indicate that the drug was present mainly in the dissolved state (molecular dispersion) $429[30]$.

430 FTIR analysis was used to study the interactions between the drug and polymer. There 431 was no evidence of strong bonds between DXI and PLGA-PEG or between NSs and the 432 polymer (Figure A.3, Supplementary Material). DXI presents a peak at $1697 \mathrm{~cm}^{-1}$ due to $433 \mathrm{C}=\mathrm{O}$ stretching, some small peaks corresponding to $\mathrm{C}-\mathrm{C}$ stretching $(1403,1461$ and 1504 $\left.434 \mathrm{~cm}^{-1}\right)$ and $\mathrm{C}-\mathrm{O}\left(1277 \mathrm{~cm}^{-1}\right)$ and finally a peak at $777 \mathrm{~cm}^{-1}$ corresponding to $\mathrm{OH}$ bending 435 [31]. PLGA-PEG exhibits intense bands at 2907 and $2950 \mathrm{~cm}^{-1}$ corresponding to the C-H 436 stretching, also present in NSs formulations. An intense peak at $1743 \mathrm{~cm}^{-1}$ is shown by 437 the polymer and the developed formulations, this corresponds to the $\mathrm{C}=\mathrm{O}$ stretching 438 vibration of the carbonyl groups present in the two monomers that form the polymer 439 matrix. Bands obtained 1077, 1199 and $1305 \mathrm{~cm}^{-1}$ in the developed formulations and in 440 the PLGA-PEG profile were attributed to stretching vibrations of the $\mathrm{OH}$ group [32]. The 441 pattern displayed by both empty and drug loaded NSs correspond to the polymer bands, 442 but their absorbance was probably increased due to the DXI present in the DXI-PLGA443 PEG NSs. It is worth to remark that neither empty nor DXI NSs showed the characteristic 444 peak corresponding to PVA (at $3300 \mathrm{~cm}^{-1}$ ), indicating an effective reduction of the surfactant amount by centrifugation process [15].

DSC profiles of DXI (Figure 2b) show a sharp endotherm corresponding with its melting transition characterized by a $\Delta \mathrm{H}=86.35 \mathrm{~J} / \mathrm{g}$ and a $\mathrm{T}_{\max }=53.06^{\circ} \mathrm{C}$, which were not detected in DXI-PLGA-PEG NSs [31]. This fact suggests that DXI formulated in PLGA-PEG NSs are in an amorphous or disordered crystalline phase of a molecular dispersion or a solid solution state in the polymer matrix [26]. These results are in accordance to those obtained by other authors [4]. The polymer presented the onset of the glass transition $\left(\mathrm{T}_{\mathrm{g}}\right)$ at 43.50 ${ }^{\circ} \mathrm{C}$, whereas the NSs presented the onset at $42.50^{\circ} \mathrm{C}$, due to drug-polymer interaction. The slight decrease of NSs $\mathrm{T}_{\mathrm{g}}$ against polymer $\mathrm{T}_{\mathrm{g}}$ has been attributed to the effect of the acidic drug due to weak interactions with PLGA [33] and [34]. PVA showed a peak at 193.55 
(a)

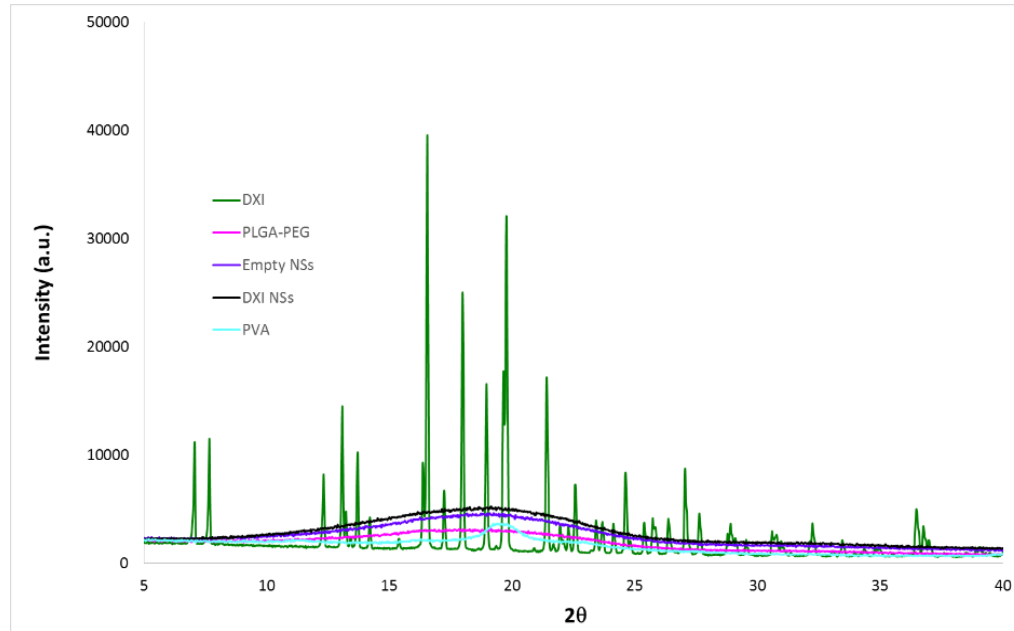

(b)

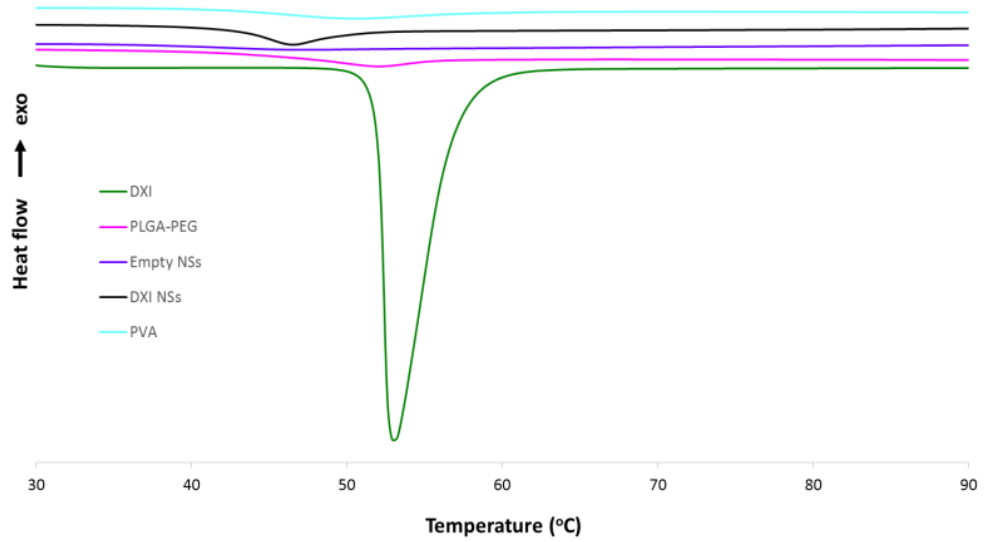

Figure 2. Physical characterization of DXI-PLGA-PEG NSs, empty PLGA-PEG NSs and NSs compound separately. (a) X-Ray diffraction patterns, (b) Differential scanning calorimetry.

\subsection{In vitro drug release}

The release profiles of free DXI and DXI loaded NSs are shown in Figure 3a. As expected, free DXI showed faster release kinetics than the drug-loading particles. After three hours, the free drug achieved $100 \%$ release, whereas after 12 hours the NSs have released $55 \%$ of the initial amount [35]. This assay confirms that NSs could release the drug at a faster rate during the first 3 hours followed by a slower diffusion (Figure 3a, triangle symbols), which would assure a prolonged effect by a slower drug release. Some authors describe that the drug can be released from PLGA matrix either via diffusion, polymer erosion or by a combination of both mechanisms. But if drug diffusion is faster than matrix degradation, drug release occurs mainly by diffusion [4] and [26]. In our case, a burst effect was observed, due to the fraction of DXI, which is absorbed or weakly bounded to the large surface area of the NSs. The second part of the profile corresponds to a sustained release behavior, where the loaded DXI slowly diffuses from the polymeric matrix to the 
477 release medium. In order to ascertain the kinetic model that better fits for DXI release, 478 data were adjusted to the most common kinetic models [36]. The most appropriate release 479 profile corresponds with a hyperbola equation. NSs $K_{d}$ was higher than the free drug, this 480 confirms the slower DXI release from the particle matrix. These results indicate that the 481 developed formulations could offer a prolonged release of DXI from the polymeric matrix 482 where it is dispersed [26].

\subsection{Ex vivo corneal and scleral permeation study}

An ex vivo corneal and scleral permeation study, comparing NSs formulation with the free DXI, was carried out for 6 hours. Results and permeation parameters are summarized in Figure $3 \mathrm{~b}$ and $3 \mathrm{c} . \mathrm{J}$ and $\mathrm{Kp}$ values in the cornea and the sclera are both similar in free 488 DXI, whereas DXI NSs present high corneal permeation and accumulation in the cornea 489 than in the sclera. This fact could be useful due to drug effect on the cornea and aqueous 490 humor. Moreover, the amount of drug released trough the cornea was higher in DXI NSs 491 than in free DXI and the opposite effect was found on the sclera. This study shows that 492 DXI NSs may deliver the drug effectively to the specified area by releasing DXI slowly 493 across the corneal tissue which would be useful in the treatment of inflammatory process 494 such as that induced by cataract surgery. $T_{L}$ corresponding with DXI NSs on the cornea 495 and sclera are smaller than free DXI; thus displaying a sustained release achieved by the 496 NSs on the studied tissues. 
(a)

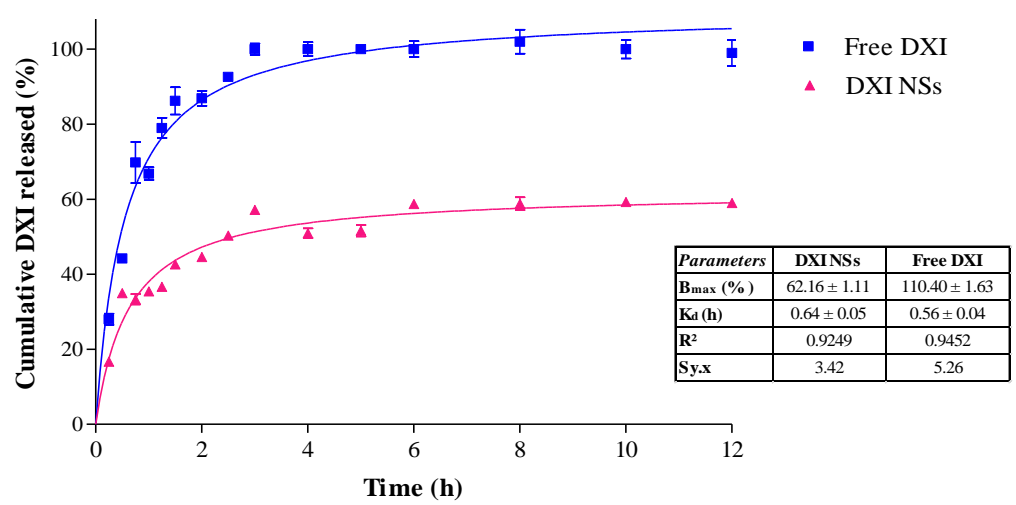

497

(b)

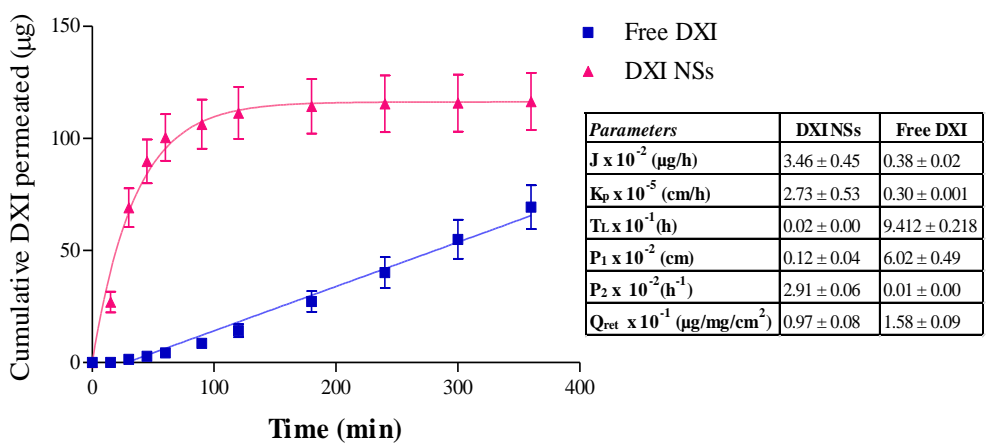

(c)

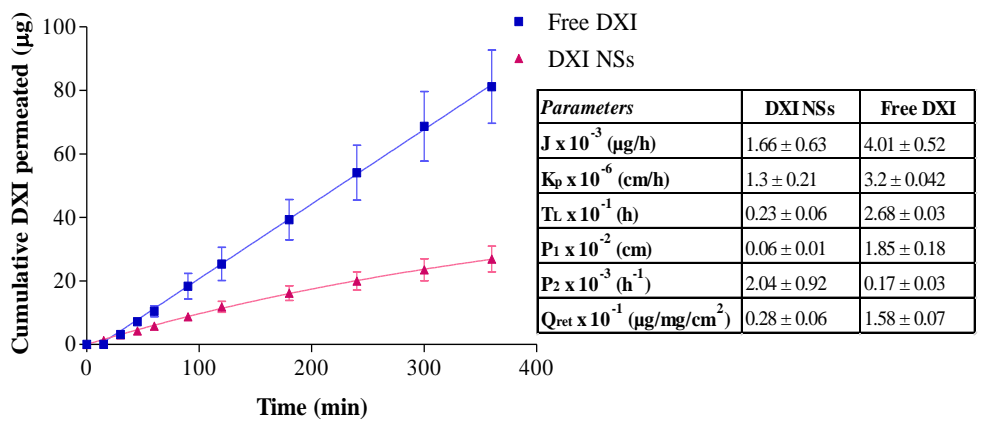

499

Figure 3. Release profiles of free DXI against DXI-PLGA-PEG NSs (n=6/group). (a) In vitro release, (b) Ex vivo corneal permeation, (c) Ex vivo scleral permeation

\subsection{Short-term stability}

Figure 4 shows the evolution of the backscattering (BS) profile of DXI loaded NSs during the first months of storage. The above graphs show instability, affecting the homogeneity of the sample (fluctuations in BS signals, lower than 10\%) in the third month of storage for $\mathrm{F}(\mathrm{A})$. The particles showed a significant decrease of the surface charge in the third month, in accordance with backscattering results (Table A.4, Supplementary Material). Due to the aggregation phenomena, $\mathrm{F}(\mathrm{B})$ was shown to be unstable at the end of the second month of storage (Fig. $4 \mathrm{~b}$ ), decreasing the ZP and increasing particle size. The limited stability of polymeric NPs in aqueous suspension is well known and these results confirm that in order to improve long-term stability, the removal of water from the solution (either by freeze drying or spray drying) is necessary [22]. Having into account 
the fact that the expiration date of collyria is limited to one month after opening the bottle, the stability of freeze dried samples, reconstituted before application, would be more suitable for ocular administration.

(a)

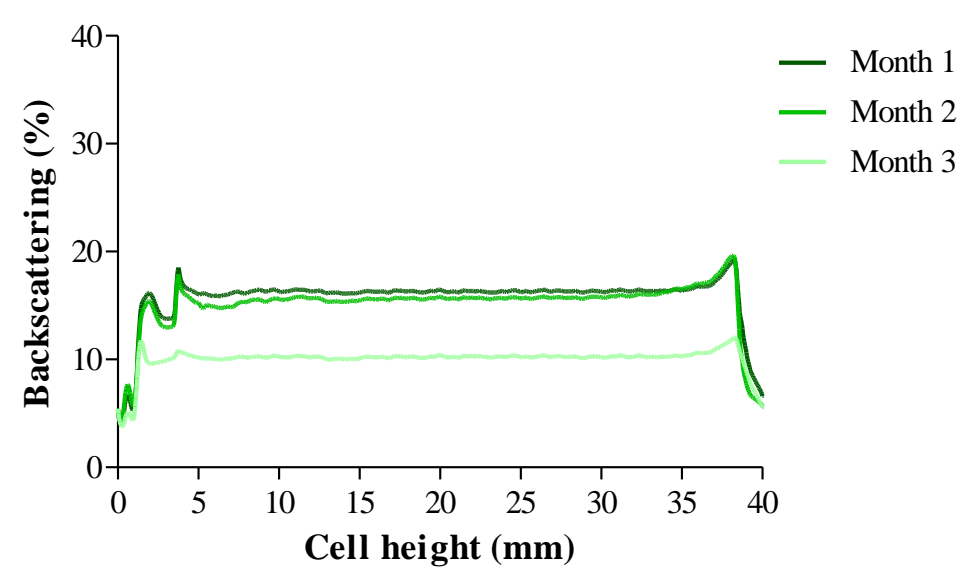

(b)

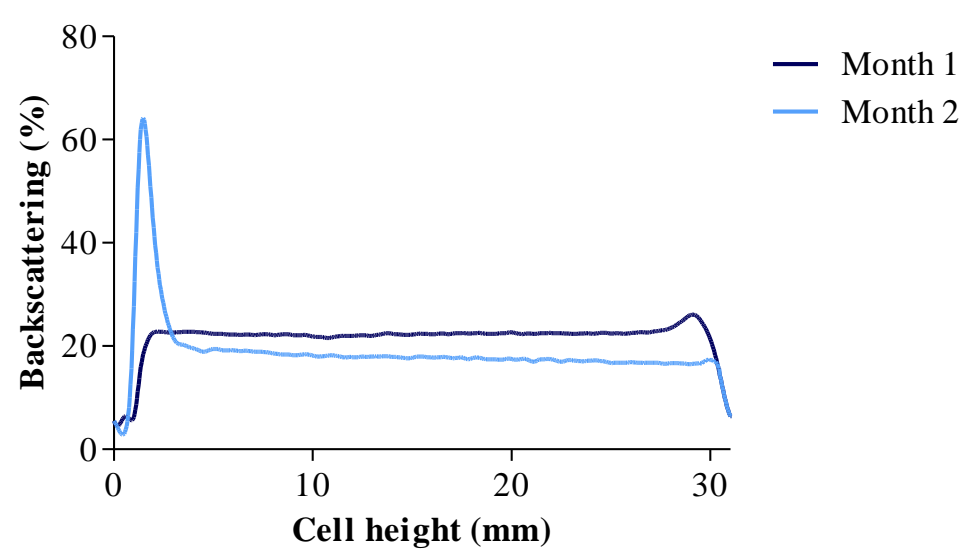

Figure 4. DXI-PLGA-PEG NSs backscattering profile. (a) F(A), (b) F(B).

\subsection{Cytotoxicity assay}

Evaluation of cell viability is important to ensure the safety of the developed NSs and avoid cell cytotoxicity. Our results demonstrate that F(A) NSs are safer, in the first $24 \mathrm{~h}$, than the free drug, in all the tested concentrations (Figure 5a). NSs with a concentration of $50 \mu \mathrm{g} / \mathrm{ml}$ slightly decreased cell viability (15\% decrease). Although cell viability of the free DXI was lower than the obtained with the F(A) NSs at $24 \mathrm{~h}$, both exhibit cell viability above $80 \%$. After $48 \mathrm{~h}$, cells exposed to free DXI showed more than $90 \%$ survival, probably due to DXI metabolism by the cytochrome P450. This could be due to metabolite formation, namely 2-[4-(2-hydroxy-2- methylpropyl)phenyl] propionic acid and 2-[3-(2-carboxypropyl)phenyl] propionic acid within $48 \mathrm{~h}$ of contact, which are not toxic for the cell [37].

The results from cell viability studies corresponding to $F(B)$ NSs are shown in Figure $5 b$. Cells exposed to the concentration of $100 \mu \mathrm{g} / \mathrm{ml}$ of NSs showed $70 \%$ cell viability in the 
first $24 \mathrm{~h}$, whereas at $48 \mathrm{~h}$ the same concentration did not show cytotoxic effects, attributed to a drug degradation mechanism. Rearding the other concentrations, NSs were safe and produced higher rates of cell survival than the free drug. No statistically significant differences were detected when comparing free drug and the NSs for F(A) and $\mathrm{F}(\mathrm{B})$.

(a)

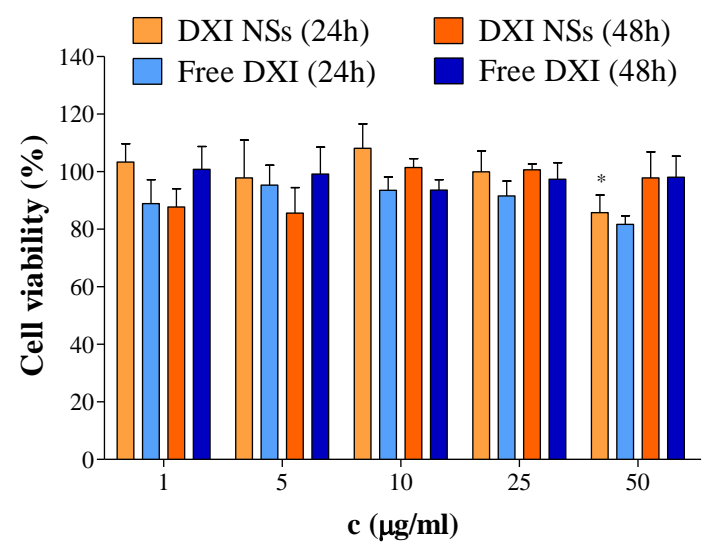

(b)

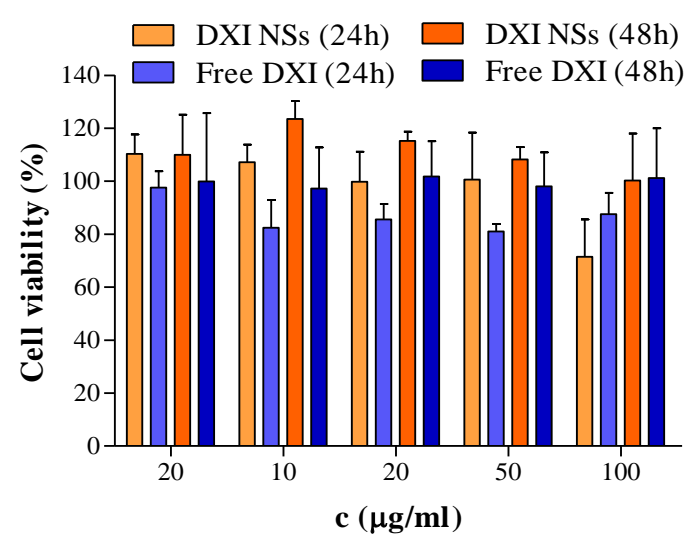

Figure 5. Alamar Blue cytotoxicity of DXI NSs against free DXI. (a) F(A) DXI-PLGAPEG NSs at different concentrations, (b) F(B) DXI-PLGA-PEG NSs at different concentrations.

Values are expressed as mean $\pm \mathrm{SD} ;{ }^{*} \mathrm{p}<0.05$, significantly lower than the same formulation at different time of exposure.

\subsection{In vitro ocular tolerance}

In vitro ocular tolerance was studied using the HET-CAM test. An addition of $0.9 \%$ saline solution to the healthy membranes produced no visual response over a five minutes period. In contrast, $1 \mathrm{M} \mathrm{NaOH}$ produced severe, hemorrhage, which increased over five minutes grading this solution as severe irritant. Application of $300 \mu 1$ of the samples (F(A), F(B) or PBS solution containing 0.5 or $1 \mathrm{mg} / \mathrm{ml} \mathrm{DXI}$ ) into the chorioallantoic membrane, revealed optimal ocular tolerance in the first 5 minutes of application (Figure A.4, Supplementary Material) [38]. OII for all tested samples show a non-irritant reaction (Table A.5, Supplementary Material). These results are in accordance to those obtained by other authors loading NSAIDs into PLGA NSs for ocular applications [15] and [39]. 
A single in vitro test could not properly mimic the entire situation in vivo, therefore, tolerance assays in male albino rabbits were performed. The OII obtained for both F(A) and F(B) and for free DXI was null (Figure A.5, Supplementary material), being the NSs classified as non-irritant (Table A.5, Supplementary Material). These results are in agreement to those obtained with the HET-CAM test, confirming the suitability of the in vitro method and the non-irritant properties of the developed formulations for ocular administration [26], [38] and [40].

\subsection{Inhibition of the inflammation}

Two studies were performed to determine the anti-inflammatory efficacy of the developed NSs, in order to confirm their usefulness for preventing and treating inflammation.

F(A) NSs prevent inflammation showing significant differences regarding positive control within the first 30 minutes after SA administration $(p<0.01)$ (Figure 6a). As described elsewhere, this correlates with the amount of drug retained in the cornea [15]. In addition, free DXI at $0.5 \mathrm{mg} / \mathrm{ml}$ also prevents inflammation compared to the control which shows significant differences after 1 hour of testing. This demonstrates that reduced DXI doses are an effective therapeutic agent for ocular inflammation. In addition, encapsulation in polymeric NSs, increases drug effect. These results show that $\mathrm{F}(\mathrm{A})$ would be adequate to prevent ocular inflammation. $F(B)$ also significantly reduced inflammation significantly after 30 minutes of application $(p<0.001)$. In general, PLGAPEG nanoparticles enhance ocular bioavailability of drugs due the especial behaviour of PEG that facilitates the drug-mucin interactions [12] and [41].

Conjunctival inflammation with significant hyperemia was induced by SA after 30 minutes of exposure. At this time, the drug was applied to the conjunctival sac and degree of inflammation was measured accurately. Formulations containing $1 \mathrm{mg} / \mathrm{ml}$ of DXI, $\mathrm{F}(\mathrm{B})$ and the corresponding free drug, reduced the inflammation faster (Figure 6b), making it adequate for high rates of inflammation which need an emergency treatment. However, F(A) demonstrated to reduce the inflammatory response more effectively than the control after 1 hour of application $(p<0.001)$.

The differences observed in degrees of inflammation and the prevention and treatment of this pathology could be related to the different absorption of NSs in healthy and inflamed tissues. This could be because the instillation of SA prior to the administration of developed formulations lead to enhanced lacrimation increasing precorneal loss and clearance of NSs [42]. F(A) would be adequate for the prevention of inflammatory injuries (e.g. cataract surgery) reducing inflammation and probably provides less adverse systemic effects than free drug or F(B) NSs [42].

The results obtained in this study are in accordance with other literature data, such as studies carried out by Buccolo et al [43] and also Musumeci et al [29] with melatonin- 
loaded PLGA-PEG, that show a higher prolonged lowering of IOP in rabbits for melatonin-PLGA-PEG nanoparticles than obtained without drug encapsulation. In these systems mucoadhesion can be related to the PEG crown which allows a better and longer interaction between the nanocarrier and the eye [44]. Similar results were obtained for acyclovir-loaded PEG-PLA nanospheres [45].

(a)

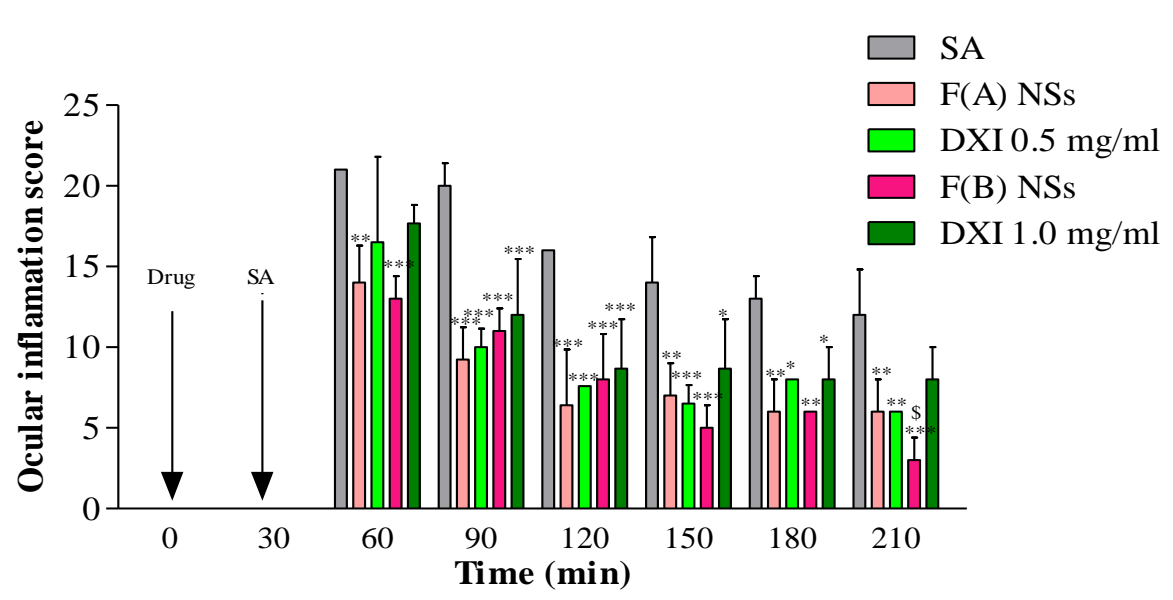

(b)

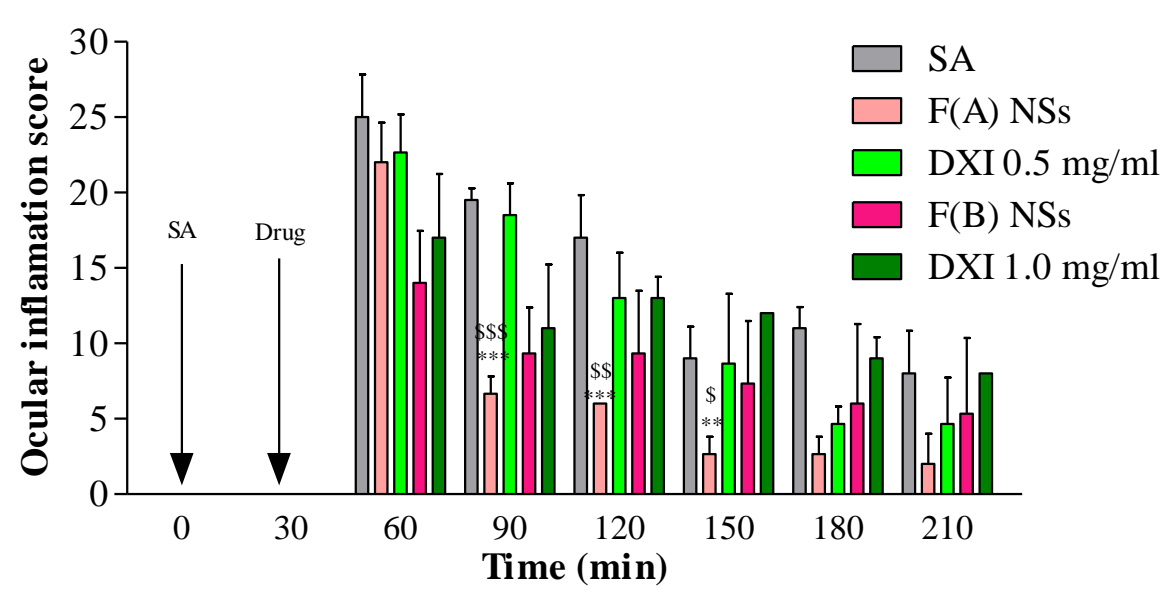

Figure 6. Comparison of ocular anti-inflammatory efficacy of $F(A), F(B)$ and the free DXI. (a) Inflammation treatment, (b) inflammation prevention.

Values are expressed as mean $\pm \mathrm{SD} ;{ }^{*} \mathrm{p}<0.05$, ${ }^{* *} \mathrm{p}<0.01$ and ${ }^{* * *} \mathrm{p}<0.001$ significantly lower than the inflammatory effect induced by $S A ;{ }^{\$}<<0.05,{ }^{\$} p<0.01$ and ${ }^{\$ \$} p<0.001$ significantly lower than the inflammatory effect induced by the corresponding free drug.

Globally, F(A) would be a sufficient treatment in the prevention of inflammation and the treatment of medium-low inflammation pathologies. In the case of rescue treatment, both slight free DXI at $1 \mathrm{mg} / \mathrm{ml}$ and $\mathrm{F}(\mathrm{B})$ would be suitable, representing the NSs an improvement in reducing corneal inflammation levels. The NSs improvement during the first hour could be due to NSs corneal preference. As demonstrated by the ocular permeation studies, free DXI is distributed and retained in the cornea and the sclera indistinctively, whereas DXI NSs provide higher drug levels on the cornea, as well a higher drug penetration to achieve aqueous humor. 


\subsection{Ocular drug bioavailability}

632 In order to elucidate NSs amount into eye structures, $\mathrm{F}(\mathrm{A})$ was administered in vivo and 633 DXI amount was quantified 2 hours after the last administration. DXI amount in the 634 cornea $(3.08 \mu \mathrm{g} / \mathrm{ml})$ was higher in comparison to every other tissue, including the sclera $635(1.28 \mu \mathrm{g} / \mathrm{ml})$. These results are in accordance to those obtained in ex vivo corneal and scleral permeation study. As reported by other authors [43], a certain amount of drug was also measured in the aqueous humor (in our case, $0.32 \mu \mathrm{g} / \mathrm{ml}$ ), but no DXI was found in the vitreous humor. These results demonstrate that DXI NSs remained retained in the first structures of the eye and released the drug slowly to inner tissues such as aqueous humor.

Moreover, it has been demonstrated that with small amount of drug, the active enantiomer loaded within NSs achieves an effective ocular anti-inflammatory activity, thus probably leading to a potential reduction in adverse effects.

\section{Conclusions}

Ocular administration for the treatment of pathological eye tissues offers the advantage of delivering the drug directly to the site of action whilst providing high drug concentration. In this study PLGA-PEG NSs were developed for topical delivery of DXI. The DoE approach shows that $\mathrm{pH}$ was one of the most influential parameters on the preparation of the nanoparticles. The optimized formulations of NSs were shown to be monodisperse $(\mathrm{PI}<0.1$ ), with a mean particle size smaller than $200 \mathrm{~nm}$, with a negative surface charge and high EE. DSC studies showed that DXI was distributed as a molecular dispersion inside the polymeric matrix. XRD showed evidence of the drug loaded within the NSs. FTIR studies showed that there was no evidence of chemical interaction or strong bond formation between the NSs compounds. F(A) NSs showed stability at $25^{\circ} \mathrm{C}$ for three months, whereas $\mathrm{F}(\mathrm{B}) \mathrm{NSs}$ showed a sedimentation process in the second month probably due to an increase in polymer and drug concentration that, in addition, contributed to their interactions. DXI in vitro release from the polymeric matrix was slower than the release of free drug. Ex vivo and in vivo studies confirmed that NSs permeate better trough corneal tissue than free DXI. The opposite effect was observed for the sclera, thus confirming that NSs were appropriate for the treatment of corneal inflammation. Cytotoxicity studies show that NSs do not reduce significantly cell viability with respect to the free drug. Both presented high survival percentages. HETCAM assay results correlate with Draize test, both showing good ocular tolerance for the developed colloidal systems. In vivo assays with $\mathrm{F}(\mathrm{A})$ showed therapeutic effects on prevention and inflammation treatment.

Our study demonstrates the advantages of using DXI-loaded PLGA nanospheres coated with PEG for prophylaxis of eye inflammation and/or for the treatment of non-severe inflammatory processes. The results obtained from the pharmacokinetic studies confirm the capacity of the developed PLGA-PEG NSs to achieve a sustained release of DXI, therefore reducing its systemic absorption and associated side effects. 
672 Acknowledgments

673

674 This work was supported by the Spanish Ministry of Science and Innovation (MAT 2014675 59134-R project). MLG, ACC, ME, MAE and ESL belong to 2014SGR-1023 and AC 676 and ME belong to 2014SGR 525. The first author, ESL, acknowledges the support of the 677 Spanish Ministry for the PhD scholarship FPI-MICINN (BES-2012-056083). We also 678 acknowledge FCT - Portuguese Foundation for Science and Technology, under the 679 project UID/AGR/04033/2013.

680

681 


\section{References}

[1] F.E. Silverstein, G. Faich, J.L. Goldstein, L.S. Simon, T. Pincus, A. Whelton, et al., Gastrointestinal Toxicity With Celecoxib vs Nonsteroidal Anti-inflammatory Drugs for Osteoarthritis and Rheumatoid Arthritis, Am. Med. Assoc. 284 (2000) 1247-1255.

[2] E.B. Souto, S. Doktorovova, E. Gonzalez-Mira, M.A. Egea, M.L. Garcia, Feasibility of lipid nanoparticles for ocular delivery of anti-inflammatory drugs, Curr. Eye Res. 35 (2010) 537-52. doi:10.3109/02713681003760168.

[3] S.T. Kaehler, W. Phleps, E. Hesse, Dexibuprofen: pharmacology, therapeutic uses and safety, Inflammopharmacology. 11 (2003) 371-383. doi:10.1017/CBO9781107415324.004.

[4] E. Vega, F. Gamisans, M.L. García, A. Chauvet, F. Lacoulonche, M.A. Egea, PLGA nanospheres for the ocular delivery of flurbiprofen: drug release and interactions, 97 (2008) 5306-5317. doi:10.1002/jps.

[5] R.C. Nagarwal, S. Kant, P.N. Singh, P. Maiti, J.K. Pandit, Polymeric nanoparticulate system: a potential approach for ocular drug delivery, J. Control. Release. 136 (2009) 2-13. doi:10.1016/j.jconrel.2008.12.018.

[6] A. Bonabello, M.R. Galmozzi, R. Canaparo, G.C. Isaia, L. Serpe, E. Muntoni, et al., Dexibuprofen (S(+)-Isomer Ibuprofen) reduces gastric damage and improves analgesic and antiinflammatory effects in rodents, Anesth. Pharmacol. 97 (2003) 402-408. doi:10.1213/01.ANE.0000073349.04610.42.

[7] W. Phleps, Overview on clinical data of dexibuprofen., Clin. Rheumatol. 20 (2001) S15-21.

[8] O. Zamani, E. Böttcher, J.D. Rieger, J. Mitterhuber, R. Hawel, S. Stallinger, et al., Comparison of safety, efficacy and tolerability of dexibuprofen and ibuprofen in the treatment of osteoarthritis of the hip or knee, Wien. Klin. Wochenschr. 126 (2014) 368-375. doi:10.1007/s00508-014-0544-2.

[9] S.A. Salem, N.M. Hwei, A. Bin Saim, C.C.K. Ho, I. Sagap, R. Singh, et al., Polylactic-co-glycolic acid mesh coated with fibrin or collagen and biological adhesive substance as a prefabricated, degradable, biocompatible, and functional scaffold for regeneration of the urinary bladder wall, J. Biomed. Mater. Res. - Part A. 101 A (2013) 2237-2247. doi:10.1002/jbm.a.34518.

[10] N. Graf, D.R. Bielenberg, N. Kolishetti, C. Muus, J. Banyard, O.C. Farokhzad, et al., $\alpha v \beta 3$ Integrin-targeted PLGA-PEG nanoparticles for enhanced anti-tumor efficacy of a Pt(IV) prodrug, ACS Nano. 6 (2012) 4530-4539.

[11] J.M. Anderson, M.S. Shive, Biodegradation and biocompatibility of PLA and PLGA microspheres, Adv. Drug Deliv. Rev. 64 (2012) 72-82. doi:10.1016/j.addr.2012.09.004.

[12] P.C. Griffiths, B. Cattoz, M.S. Ibrahim, J.C. Anuonye, Probing the interaction of nanoparticles with mucin for drug delivery applications using dynamic light scattering, Eur. J. Pharm. Biopharm. 97 (2015) 218-222. doi:10.1016/j.ejpb.2015.05.004.

[13] S. Akhter, F. Ramazani, M.Z. Ahmad, F.J. Ahmad, Z. Rahman, A. Bhatnagar, et al., Ocular pharmacoscintigraphic and aqueous humoral drug availability of ganciclovir-loaded mucoadhesive nanoparticles in rabbits, Eur. J. Nanomedicine. 
5 (2013) 159-167. doi:10.1515/ejnm-2013-0012.

[14] N.M. Khalil, T.C.F. do Nascimento, D.M. Casa, L.F. Dalmolin, A.C. de Mattos, I. Hoss, et al., Pharmacokinetics of curcumin-loaded PLGA and PLGA-PEG blend nanoparticles after oral administration in rats, Colloids Surfaces B Biointerfaces. 101 (2013) 353-360. doi:10.1016/j.colsurfb.2012.06.024.

[15] G. Abrego, H.L. Alvarado, M.A. Egea, E. Gonzalez-Mira, A.C. Calpena, M.L. Garcia, Design of nanosuspensions and freeze-dried PLGA nanoparticles as a novel approach for ophthalmic delivery of pranoprofen., J. Pharm. Sci. 103 (2014) 3153-64. doi:10.1002/jps.24101.

[16] J.F. Fangueiro, T. Andreani, M. a. Egea, M.L. Garcia, S.B. Souto, A.M. Silva, et al., Design of cationic lipid nanoparticles for ocular delivery: Development, characterization and cytotoxicity, Int. J. Pharm. 461 (2014) 64-73. doi:10.1016/j.ijpharm.2013.11.025.

[17] D. Cun, D.K. Jensen, M.J. Maltesen, M. Bunker, P. Whiteside, D. Scurr, et al., High loading efficiency and sustained release of siRNA encapsulated in PLGA nanoparticles: Quality by design optimization and characterization, Eur. J. Pharm. Biopharm. 77 (2011) 26-35. doi:10.1016/j.ejpb.2010.11.008.

[18] E. Gonzalez-Mira, S. Nikolic, A.C. Calpena, M.A. Egea, E.B. Souto, M.L. García, Improved and safe transcorneal delivery of flurbiprofen by NLC and NLC-based hydrogels, J. Pharm. Sci. 101 (2012) 707-725. doi:10.1002/jps.

[19] M. Ganesan, K.S. Rauthan, Y. Pandey, P. Tripathi, Determination of Ibuprofen in Human Plasma With Minimal Sample, Int. J. Pharm. Sci. Res. 1 (2010) 120-127.

[20] J.-X. Wang, X. Sun, Z.-R. Zhang, Enhanced brain targeting by synthesis of $3^{\prime}, 5^{\prime}-$ dioctanoyl-5-fluoro-2'-deoxyuridine and incorporation into solid lipid nanoparticles, Eur. J. Pharm. Biopharm. 54 (2002) 285-290. doi:10.1016/S09396411(02)00083-8.

[21] M. Teixeira, M.J. Alonso, M.M.M. Pinto, C.M. Barbosa, Development and characterization of PLGA nanospheres and nanocapsules containing xanthone and 3-methoxyxanthone, Eur. J. Pharm. Biopharm. 59 (2005) 491-500. doi:10.1016/j.ejpb.2004.09.002.

[22] G. Abrego, H. Alvarado, E.B. Souto, B. Guevara, L. Halbaut, A. Parra, et al., Biopharmaceutical profile of pranoprofen-loaded PLGA nanoparticles containing hydrogels for ocular administration, Eur. J. Pharm. Biopharm. 231 (2015) 1-10. doi:10.1016/j.ejpb.2015.01.026.

[23] S. Doktorovová, D.L. Santos, I. Costa, T. Andreani, E.B. Souto, A.M. Silva, Cationic solid lipid nanoparticles interfere with the activity of antioxidant enzymes in hepatocellular carcinoma cells, Int. J. Pharm. 471 (2014) 18-27. doi:10.1016/j.ijpharm.2014.05.011.

[24] M. Warren, K. Atkinson, S. Steer, INVITTOX: The ERGATT/FRAME data bank of in vitro techniques in toxicology, Toxicol. Vitr. 4 (1990) 707-710. doi:10.1016/0887-2333(90)90148-M.

[25] D. Jírová, K. Kejlová, S. Janoušek, H. Bendová, M. Malý, H. Kolářová, et al., Eye irritation hazard of chemicals and formulations assessed by methods in vitro, Neuroendocrinol. Lett. 35 (2014) 133-140.

[26] E. Vega, M.A. Egea, A.C. Calpena, M. Espina, M.L. García, Role of hydroxypropyl- $\beta$-cyclodextrin on freeze-dried and gamma-irradiated PLGA and 
PLGA - PEG diblock copolymer nanospheres for ophthalmic flurbiprofen delivery, Int. J. Nanomedicine. 7 (2012) 1357-1371.

[27] C. Bucolo, F. Drago, S. Salomone, Ocular drug delivery: A clue from nanotechnology, Front. Pharmacol. 3 (2012) 1-3. doi:10.3389/fphar.2012.00188.

[28] G. Ma, C. Zhang, L. Zhang, H. Sun, C. Song, C. Wang, et al., Doxorubicin-loaded micelles based on multiarm star-shaped PLGA-PEG block copolymers: influence of arm numbers on drug delivery, J. Mater. Sci. Mater. Med. 27 (2016) 1-15. doi:10.1007/s10856-015-5610-4.

[29] T. Musumeci, C. Bucolo, C. Carbone, R. Pignatello, F. Drago, G. Puglisi, Polymeric nanoparticles augment the ocular hypotensive effect of melatonin in rabbits, Int. J. Pharm. 440 (2013) 135-140. doi:10.1016/j.ijpharm.2012.10.014.

[30] E. Vega, M.A. Egea, M.L. Garduño-Ramírez, M.L. Garcia, E. Sánchez, M. Espina, et al., Flurbiprofen PLGA-PEG nanospheres: role of hydroxy- $\beta$-cyclodextrin on ex vivo human skin permeation and in vivo topical anti-inflammatory efficacy, Colloids Surfaces B Biointerfaces. $110 \quad$ (2013) 339-346. doi:10.1016/j.colsurfb.2013.04.045.

[31] B.M. El-Houssieny, E.Z. El-Dein, H.M. El-Messiry, Enhancement of solubility of dexibuprofen applying mixed hydrotropic solubilization technique, Drug Discov. Ther. 8 (2014) 178-184. doi:10.5582/ddt.2014.01019.

[32] R. Singh, P. Kesharwani, N.K. Mehra, S. Singh, S. Banerjee, N.K. Jain, Development and characterization of folate anchored Saquinavir entrapped PLGA nanoparticles for anti-tumor activity, Drug Dev. Ind. Pharm. 41 (2015) 1888-1901. doi:10.3109/03639045.2015.1019355.

[33] F. Alexis, Factors affecting the degradation and drug-release mechanism of poly(lactic acid) and poly[(lactic acid)-co-(glycolic acid)], Polym. Int. 54 (2005) 36-46. doi:10.1002/pi.1697.

[34] M. Miyajima, A. Koshika, J. Okada, M. Ikeda, Mechanism of drug release from poly(l-lactic acid) matrix containing acidic or neutral drugs, J. Control. Release. 60 (1999) 199-209. doi:10.1016/S0168-3659(99)00083-8.

[35] S. Muralidharan, S.N. Meyyanathan, K. Krishnaraj, S. Rajan, Development of oral Sustained release dosage form for low melting chiral compound Dexibuprofen and it's in vitro-in vivo evaluation, Int. J. Drug Deliv. 3 (2011) 492-502.

[36] P. Costa, J.M. Sousa Lobo, Modeling and comparison of dissolution profiles, Eur. J. Pharm. Sci. 13 (2001) 123-133. doi:10.1016/S0928-0987(01)00095-1.

[37] N.M. Davies, Clinical Pharmacokinetics of Ibuprofen The First 30 Years, Drug Diisposition. 34 (1998) 101-154.

[38] A.M.D. Nóbrega, E.N. Alves, R.D.F. Presgrave, R.N. Costa, I.F. Delgado, Determination of eye irritation potential of low-irritant products: comparison of in vitro results with the in vivo draize rabbit test, Brazilian Arch. Biol. Technol. 55 (2012) 381-388. http://www.scielo.br/scielo.php?pid=S151689132012000300008\&script=sci_arttext.

[39] J. Araújo, E. Vega, C. Lopes, M.A. Egea, M.L. Garcia, E.B. Souto, Effect of polymer viscosity on physicochemical properties and ocular tolerance of FBloaded PLGA nanospheres, Colloids Surf. B. Biointerfaces. 72 (2009) 48-56. doi:10.1016/j.colsurfb.2009.03.028. 
[40] B. McKenzie, G. Kay, K.H. Matthews, R.M. Knott, D. Cairns, The hen's egg chorioallantoic membrane (HET-CAM) test to predict the ophthalmic irritation potential of a cysteamine-containing gel: quantification using Photoshop ${ }^{\circledR}$ and ImageJ, Int. J. Pharm. 490 (2015) 1-8. doi:10.1016/j.jphharm.2015.05.023.

[41] T. Andreani, L. Miziara, E.N. Lorenzón, A.L.R. De Souza, C.P. Kiill, J.F. Fangueiro, et al., Effect of mucoadhesive polymers on the in vitro performance of insulin-loaded silica nanoparticles: Interactions with mucin and biomembrane models, Eur. J. Pharm. Biopharm. 93 (2015) 118-126. doi:10.1016/j.ejpb.2015.03.027.

[42] A. Vasconcelos, E. Vega, Y. Pérez, M.J. Gómara, M.L. García, I. Haro, Conjugation of cell-penetrating peptides with poly(lactic-co-glycolic acid)polyethylene glycol nanoparticles improves ocular drug delivery, Int. J. Nanomedicine. 10 (2015) 609-631. doi:10.2147/IJN.S71198.

[43] C. Bucolo, A. Maltese, G. Puglisi, R. Pignatello, Enhanced ocular antiinflammatory activity of ibuprofen carried by an Eudragit RS100 nanoparticle suspension, Opthalmic Reseacrh. 34 (2002) 319-323. doi:10.1159/000065608.

[44] A. Ludwig, The use of mucoadhesive polymers in ocular drug delivery, Adv. Drug Deliv. Rev. 57 (2005) 1595-1639. doi:10.1016/j.addr.2005.07.005.

[45] C. Giannavola, C. Bucolo, A. Maltese, D. Paolino, M.A. Vandelli, G. Puglisi, et al., Influence of preparation conditions on acyclovir-loaded poly-d,l-lactic acid nanospheres and effect of PEG coating on ocular drug bioavailability, Pharm. Res. 20 (2003) 584-590. doi:10.1023/A:1023290514575. 